\title{
Manejo laparoscópico de la dehiscencia anastomótica en cirugía colorrectal
}

\author{
Mariano Laporte, Mariana Matzner Perfumo, Jose Piatti, Laura Mattioni, Alejandro Canelas, \\ Maximiliano Bun, Nicolás Rotholtz \\ Sector Cirugía Colorrectal, Servicio Cirugía General, Hospital Alemán de Buenos Aires. CABA, Argentina.
}

\begin{abstract}
RESUMEN
Introducción: En los últimos años ha habido una gran difusión de la cirugía laparoscópica para el manejo de la patología colorrectal. La dehiscencia anastomótica es una de las complicaciones más graves, con una elevada morbi-mortalidad. La reoperación por vía laparoscópica podría ser una opción válida para tratar esta complicación, manteniendo ciertos beneficios del abordaje miniinvasivo.

Objetivos: Evaluar la factibilidad y seguridad del abordaje laparoscópico en el manejo de la dehiscencia anastomótica en cirugía colorrectal y en forma secundaria comparar los resultados con la reoperación por vía convencional.

Materiales y Método: Se analizó una serie retrospectiva, completada en forma prospectiva, se incluyeron 1693 pacientes (junio 2000 - septiembre 2018). Los pacientes que fueron reoperados por dehiscencia anastomótica se dividieron en dos grupos según el abordaje de la reoperación: laparoscópico (Grupo 1, G1) y laparotómico (Grupo 2, G2). Se compararon ambos grupos teniendo en cuenta factores demográficos, estadía hospitalaria, complicaciones, morbilidad y mortalidad. Las complicaciones se estratificaron según la clasificación de Dindo y Clavien, y se tuvieron en cuenta las más graves (categorías 3, 4 y 5). Para el análisis estadístico se utilizó el T student y chi cuadrado.

Resultados: Ciento seis $(6,26 \%)$ pacientes fueron reoperados por dehiscencia anastomótica. Ochenta y cinco $(80 \%)$ fueron incluidos en el grupo 1 y $21(20 \%)$ en el grupo 2. La única diferencia demográfica entre ambos grupos fue una mayor cantidad de pacientes obesos en el grupo laparoscópico (G1: 17 (20\%) vs. G2: 0, p: 0,02). Hubo una tendencia hacia un intervalo menor entre la cirugía inicial y la reexploración, pero sin diferencias estadísticamente significativas $(5,18$ días vs. 6,23 días, p: 0,22). En 84 (79\%) la conducta quirúrgica fue lavado y confección de ostomía proximal de protección (G1: 74 vs. G2: 10, p: 0,001). El desmonte de la anastomosis y la confección de ostomía terminal debió realizarse en 8 pacientes (G1: 4 vs G2: 4, p: 0,02). Nueve pacientes en $\mathrm{G} 1$ y 3 pacientes en $\mathrm{G} 2$ requirieron más de una cirugía ( $\mathrm{p}: 0,63)$. Las complicaciones fueron similares entre ambos grupos, sólo se incluyeron los grados 3, 4 y 5 (G1: 21,2\% vs G2: 28,6\% p: 0,34). El promedio de estadía hospitalaria disminuyó con el abordaje laparoscópico (10,71 días vs. 11,57 días, p: 0,66), a pesar de que no hubo
\end{abstract} diferencia estadística entre ambos grupos.

Conclusiones: La reintervención laparoscópica es un tratamiento válido y seguro para el manejo de la dehiscencia anastomótica en cirugía laparoscópica colorrectal.

Palabras clave: Cirugía Colorrectal Laparoscópica; Reoperación; Dehiscencia Anastomótica

\begin{abstract}
Introduction: In recent years there has been a great diffusion of laparoscopic surgery for the management of colorectal pathology. Anastomotic dehiscence is one of the most serious complications, with high morbidity and mortality. Laparoscopic reoperation could be a valid option to treat this complication, maintaining certain benefits of the minimally invasive approach. Objectives: To evaluate the viability and safety of the laparoscopic approach in the management of anastomotic dehiscence in colorectal surgery and as a secondary end point to compare the results with those of reoperation by conventional
\end{abstract} approach.

Material and Methods: A series of 1693 patients that underwent laparoscopic colorectal surgery was analyzed, from a prospective database (June 2000 - September 2018). Patients were divided into two groups according to the approach performed in the reoperative surgery: laparoscopy (G 1) or laparotomy (G 2). Demographic data, hospital stay, type of complication, morbidity and mortality were analyzed. Dindo-Clavien classification was used to stratify postoperative complications and only categories 3, 4 and 5 were included. Data were statistically analyzed with Student's t test and chisquare test.

Results: A hundred six patients (6.26\%) were reoperated because of AL, 85 (80\%) by laparoscopy and 21 (20\%) by conventional surgery. The only demographic difference between both groups was that more obese patients were included in G1 (G1: 17, 20\% vs. G2: 0, $p=0.02)$. Interval of time between surgeries was lower in G1 without statistical difference (5.18 vs. 6.23 days, $p=0.22$ ). In 84 patients (79\%) abdominal lavage and loop ostomy was performed (G1: 74 vs. G2: 10, $p=0.001$ ). Anastomosis takedown was required in 8 patients (G1: 4 vs. G2: 4, $p=0.02$ ). 9 patients in $G 1$ and 3 in G2 needed more than one reexploration $(p=0.63)$. Postoperative complications were similar in both groups, grades 3,4 and 5 were included (G1: $21,2 \%$ vs. G2: $28.6 \%, p=0.34$ ). In average hospital stay was decreased in G1 (10.7 vs. 11.6 days, $p=0.66)$, without statistical difference.

Conclusion: Laparoscopic reintervention can be a safe treatment for anastomotic leakage after laparoscopic colorectal surgery.

Key words: Colorectal Laparoscopic Surgery; Reoperative Surgery; Anastomotic Leakage

\section{INTRODUCCIÓN}

Los autores declaran ausencia de conflictos de interés.

Mariano Laporte

drmarianolaporte@gmail.com

Recibido: septiembre de 2019. Aceptado: octubre de 2019.
La cirugía laparoscópica de patología colorrectal ha mostrado los beneficios del abordaje miniinvasivo, entre los que destacan menor íleo postoperatorio y menor dolor 
postoperatorio lo que se traduce en una recuperación más rápida y menor estadía hospitalaria. Las incisiones más pequeñas y la menor tasa de infecciones de herida impactan directamente en una menor tasa de complicaciones parietales. ${ }^{1}$

Sin embargo, la tasa de complicaciones graves, como la dehiscencia anastomótica, no disminuye con la cirugía laparoscópica y se mantiene en valores alrededor del $5 \%$ independientemente del tipo de abordaje. ${ }^{2-5}$

Las ventajas de la reoperación por vía laparoscópica inicialmente fueron descritas para otro tipo de patología, complicaciones de la cirugía biliar y bariátrica. En los últimos años se han publicado varios artículos sobre las ventajas del abordaje laparoscópico para el manejo de la dehiscencia anastomótica en patología colorrectal. ${ }^{5-17}$

El objetivo del presente trabajo es evaluar la seguridad y factibilidad del abordaje miniinvasivo para el tratamiento de la dehiscencia anastomótica en pacientes que habían sido sometidos a cirugía colorrectal laparoscópica, realizando una comparación con aquellos pacientes reoperados por vía convencional.

\section{MATERIAL Y MÉTODOS}

Se realizó un análisis retrospectivo de una base de datos completada en forma prospectiva que incluye a todos los pacientes operados de patología colorrectal por vía laparoscópica, entre junio 2000 y septiembre 2018, en el Hospital Alemán de Buenos Aires. Se incluyeron en el presente estudio los pacientes que fueron reoperados por dehiscencia anastomótica, se excluyeron aquellos que respondieron favorablemente al tratamiento médico y/o percutáneo. Se conformaron dos grupos dependiendo la vía de abordaje de la reoperación. En el grupo 1 (G1) se incluyeron aquellos reoperados por vía laparoscópica y en el grupo 2 (G2) se incluyeron los que fueron reoperados en forma convencional. Se realizó un análisis comparativo de ambos grupos teniendo en cuenta factores demográficos, tipo de cirugía realizada, estadía hospitalaria, días entre operación y reoperación, morbilidad y mortalidad postoperatoria. Se definió obesidad como aquellos pacientes con un IMC superior a 30. La decisión del abordaje utilizado en cada caso dependió del criterio del cirujano actuante. Los pacientes que requirieron conversión de la segunda cirugía fueron mantenidos en el grupo laparoscópico, realizando un análisis de "intención de tratar".

\section{Técnica quirúrgica}

En todos los casos la cirugía inicial fue laparoscópica, de patología colorrectal. Las reoperaciones fueron realizadas por el mismo equipo quirúrgico o los cirujanos de guardia. En el caso de reoperaciones por vía laparoscópica se utilizaron las mismas incisiones de los trocares que habían sido realizadas en la primera cirugía. El primer trocar se introdujo según técnica abierta. En todos los casos cuando se realizó una ostomía proximal debido a una dehiscencia anastomótica se colocó un drenaje ofrecido a la anastomosis, que se extrajo por contra abertura a través de un orificio de trocar. En los pacientes que fueron reoperados por vía convencional se realizó una incisión mediana.

$\mathrm{El}$ análisis estadístico se realizó por medio utilizando $\mathrm{T}$ de student, chi cuadrado y prueba de Fischer. Se consideró p menor a 0,05 como estadísticamente significativa.

\section{RESULTADOS}

En el período comprendido entre junio 2000 y septiembre 2018, se realizaron 1693 procedimientos laparoscópicos. Ciento sesenta y uno $(9,5 \%)$ requirieron una reintervención por alguna complicación durante el primer mes del postoperatorio. Ciento seis $(6,26 \%)$ fueron reoperados por presentar dehiscencia anastomótica. Ochenta y cinco $(80,2 \%)$ fueron reoperados por vía laparoscópica y se incluyeron en el grupo 1 (G1). Veintiuno (20\%) se incluyeron en G2. En la tabla 1 se evidencian las características demográficas de ambos grupos. La única diferencia demográfica consistió en la cantidad de pacientes obesos, con índice de masa corporal superior a 30, en G1 (17 vs. $0, \mathrm{p}: 0,02)$.

En ambos grupos la cirugía inicial más frecuente era la colectomía izquierda/sigmoidectomía. En la tabla 1 se pueden objetivar el tipo de cirugía inicial en ambos grupos, sin existir diferencias estadísticamente significativas. 19 cirugías se habían convertido inicialmente, 11 fueron reoperados en forma convencional y 8 por vía laparoscópica (p: 0,005), 3 de los cuales requirieron conversión.

El intervalo entre operación y reoperación fue menor en G1 (5,18 vs. 6,23 días p:0,22), si bien no hubo diferencias estadísticamente significativas. Las conductas quirúrgicas de la reexploración se pueden objetivar en la tabla 2 , siendo la más frecuente el lavado, drenaje y la confección de una ostomía, realizándose en el $79 \%$ de los pacientes que fueron reintervenidos. Dicha conducta fue más frecuente en G1 (74 vs 10, p: 0,001). Se requirió desmontar los cabos y confeccionar una ostomía terminal en 8 pacientes (G1: 4 vs G2: 4, p: 0,02). Nueve pacientes en G1 y 3 pacientes en $\mathrm{G} 2$ requirieron más de una cirugía (p: 0,63). La tasa global de conversión de G1 fue de 8,23\% (7/85).

Las complicaciones de las reexploraciones se pueden objetivar en la tabla 2. La tasa de complicaciones mayores a 3, utilizando la clasificación de Dindo y Clavien, fue del $21,2 \%$ en $\mathrm{G} 1$ y $28,6 \%$ en $\mathrm{G} 2$, sin evidenciar diferencias estadísticamente significativas en ambos grupos (p: 0,34). La mortalidad global de la serie fue del 6,6\% y fue similar 
TABLA 1: FACTORES DEMOGRÁFICOS Y CIRUGÍAS REALIZADAS

\begin{tabular}{|c|c|c|c|c|}
\hline & & G1 & G2 & $\mathrm{p}$ \\
\hline Sexo masculino & femenino & $66 / 19$ & $15 / 6$ & 0,5 \\
\hline & & & & 0,8 \\
\hline Cirugías previas & & $44(51)$ & $14(66)$ & 0,2 \\
\hline & & & & 0,1 \\
\hline $\mathrm{BMI}>30, \mathrm{n}(\%)$ & & $17(20)$ & 0 & 0,00 \\
\hline & & & & 2 \\
\hline Edad, media (ra & go) & 58,9 & 64,3 & 0,1 \\
\hline & & $(28-83)$ & $(25-86)$ & 4 \\
\hline & 1 & $16(18,8)$ & $1(4,7)$ & 0,1 \\
\hline & & & & 2 \\
\hline & ॥ & $56(65,8)$ & $14(66,6)$ & 0,9 \\
\hline ASA, n (\%) & & & & 4 \\
\hline & III & $13(15,3)$ & $5(23,8)$ & 0,3 \\
\hline & & & & 52 \\
\hline Patología Benig & a/maligna, $n$ & $26 / 59$ & $8 / 13$ & 0,5 \\
\hline & & & & 09 \\
\hline & Colectomía derecha & $15(17)$ & $6(28,6)$ & 0,2 \\
\hline & & & & 61 \\
\hline Procedimiento & Colectomía izquierda/sigmoidectomía & $43(50,6)$ & $7(33,3)$ & 0,1 \\
\hline inicial, n (\%) & & & & 56 \\
\hline & Resección anterior & $11(13)$ & $3(14,3)$ & 0,8 \\
\hline & & & & 7 \\
\hline Conversión, n (9 & & $8(9,4)$ & $11(52,4)$ & 0,0 \\
\hline & & & & 05 \\
\hline
\end{tabular}

TABLA 2: CONDUCTA REALIZADA EN LA REINTERVENCIÓN Y COMPLICACIONES POSTOPERATORIAS

\begin{tabular}{|c|c|c|c|c|}
\hline \multirow{2}{*}{\multicolumn{2}{|c|}{ Días entre operación y reoperación, media (rango) }} & G1 & G2 & $p$ \\
\hline & & $5,2(1-18)$ & $6,2(2-14)$ & 0,22 \\
\hline \multirow{2}{*}{ Conducta en la reoperación, n (\%) } & Lavado, drenaje e ileostomía & $72(84,7)$ & $10(47,6)$ & 0,001 \\
\hline & Abocamiento de cabos & $5(5,9)$ & $3(14,3)$ & 0,36 \\
\hline $\begin{array}{l}\text { Estadía hospitalaria, días, } \\
\text { media (rango) }\end{array}$ & & $10,7(1-35)$ & $11,5(2-34)$ & 0,66 \\
\hline \multirow{5}{*}{$\begin{array}{l}\text { Complicaciones } \\
\text { postoperatorias } \\
\mathrm{n}(\%)\end{array}$} & IIla & $6(7)$ & $1(4,7)$ & 0,7 \\
\hline & IIIb & $2(2,3)$ & 0 & 0,47 \\
\hline & IVa & $6(7)$ & $2(9,5)$ & 0,72 \\
\hline & V & $4(4,6)$ & $3(14,2)$ & 0,99 \\
\hline & Total & $18(21,2)$ & $6(28,6)$ & 0,8 \\
\hline Eventración, n (\%) & & $11(13)$ & $4(19)$ & 0,47 \\
\hline
\end{tabular}


TABLA 3: ANÁLISIS MULTIVARIADO

\begin{tabular}{|c|c|c|c|c|}
\hline$x$ & Coef. & $p$ & [95\% Conf. & Interval] \\
\hline Edad & -.0272643 & 0.474 & -.1019261 & .0473975 \\
\hline IMC $>30$ & .4071577 & 0.050 & .0002535 & .8140618 \\
\hline intervalo entre cirugías & -.2638386 & 0.123 & -.5988622 & .0711851 \\
\hline Estadía hospitalaria & .0263634 & 0.766 & -.1474701 & .2001968 \\
\hline nroreop & 214.136 & 0.345 & -2.307 .081 & 65.898 \\
\hline lavado y drenaje & 4.269.728 & 0.020 & .6646297 & 7.874 .826 \\
\hline eventración pop & .413312 & 0.771 & -2.369 .162 & 3.195 .786 \\
\hline sexo & -1.312 .536 & 0.366 & -4.159 .688 & 1.534 .616 \\
\hline patología maligna & 1.840 .062 & 0.205 & -1.003 .159 & 4.683 .283 \\
\hline cirugías previas & -2.210 .281 & 0.085 & -4.725 .142 & .3045801 \\
\hline asa1 & 2.872 .536 & 0.234 & -1.857 .031 & 7.602 .103 \\
\hline asa2 & -.0008189 & 1.000 & -3.147 .038 & 31.454 \\
\hline tipo de cirugia & -.1048079 & 0.894 & -1.643 .633 & 1.434 .017 \\
\hline conversión & -1.248 .166 & 0.422 & -4.292 .633 & 1.796 .302 \\
\hline alta entre cirugías & 2.703 .375 & 0.163 & -109.726 & 650.401 \\
\hline
\end{tabular}

en ambos grupos (G1: 4,6\% vs G2:14,2\%, p: 0,99).

$\mathrm{El}$ promedio de internación hospitalaria fue menor en G1 (10,7 vs. 11,6 días, p: 0,66) si bien no hubo diferencias estadísticamente significativas.

No hubo diferencias significativas en cuanto a las eventraciones postoperatorias (G1: $13 \%$ vs. G2: 19\%, p: 0,47).

En el análisis multivariado los únicos valores que se mantuvieron con diferencias estadísticamente significativas entre ambos grupos fueron la obesidad y la conducta quirúrgica (lavado, drenaje y ostomía proximal), ambos más frecuentes en G1 (tabla 3).

\section{DISCUSIÓN}

La dehiscencia de anastomosis es la complicación más grave de la cirugía colorrectal, independientemente de la vía de abordaje la tasa ronda alrededor del $5 \%$, pero puede elevarse en determinadas situaciones a cifras cercanas al $25 \%$. En nuestra serie la tasa global de reoperaciones por dehiscencia es del 6,26\%, valor acorde a las descriptas. ${ }^{7,9,18}$ La importancia de esta complicación radica en que la mortalidad posterior a una dehiscencia anastomótica puede ascender el 30\%, dependiendo de múltiples factores. ${ }^{19}$ Adicionalmente impacta en los resultados oncológicos, incrementando la recurrencia local con impacto directo en la sobrevida a largo plazo, período libre de enfermedad y sobrevida global. ${ }^{20} \mathrm{El}$ impacto económico tampoco es despreciable, Turrentine ${ }^{21}$ describió que los gastos prácticamente se duplican cuando se presenta esta complicación (U\$D 16085 vs. U\$D 30409, p < 0,0001).

Hace un tiempo existía cierta controversia sobre la factibilidad del abordaje laparoscópico en pacientes con peritonitis, debido al potencial riesgo de endotoxemia motivado por el aumento de la presión intraabdominal por el neumoperitoneo. Sin embargo, posteriormente se pudo probar que es segura la utilización de la cirugía miniinvasiva en pacientes con peritonitis generalizadas. ${ }^{22-26}$

Durante los últimos años ha aumentado el número de publicaciones a nivel nacional e internacional que describen la utilidad del abordaje miniinvasivo para el manejo de complicaciones de patología colorrectal. ${ }^{5-17} \mathrm{Si}$ bien la mayoría son series retrospectivas parecería lógico pensar que de esta forma se mantendrían ciertos beneficios del abordaje laparoscópico. En la literatura, destaca una revisión publicada por Chang y colaboradores ${ }^{8}$ en la que se incluyen 11 series, con un total de 118 pacientes reoperados por vía laparoscópica. Los autores concluyeron que la reintervención por vía laparoscópica es segura y efectiva para el manejo de las complicaciones de cirugía colorrectal laparoscópica. En el mismo se incluye el trabajo 
publicado por nuestro grupo de trabajo en el año $2009,{ }^{27}$ que analiza una serie de total 510 pacientes sometidos a cirugía colorrectal laparoscópica, en el cual se realizó una comparación de 17 pacientes reoperados por vía miniinvasiva con 10 reoperados en forma convencional.

Si bien no hubo diferencias estadísticamente significativas en la mayoría de las características demográficas de ambos grupos, se vio una mayor tendencia a la reoperación por vía laparoscópica cuando la dehiscencia se presentó en pacientes obesos (IMC mayor a 30). Es conocida la ventaja del abordaje laparoscópico en pacientes obesos, permitiendo una adecuada visualización de toda la cavidad. ${ }^{28}$ Por otra parte, en caso de ser operado por vía convencional sin dudas requeriría una incisión de gran tamano debido a la contextura física del paciente. Esto podría ser el motivo de la mayor tendencia a reoperar pacientes obesos por esta vía. Los fundamentos de la reexploración laparoscópica en pacientes obesos que habían sido operados de patología colorrectal son escasos. Sin embargo, las ventajas de la reexploración por vía laparoscópica se pueden objetivar claramente en los pacientes complicados de cirugía bariátrica.

Es importante destacar que la reoperación precoz se asocia a mejor recuperación postoperatoria. ${ }^{14,18}$ En nuestra serie se pudo evidenciar en promedio un intervalo menor entre operación y reoperación cuando se realizó el abordaje laparoscópico, a pesar de que no hubo diferencias estadísticamente significativas. Esto podría deberse a un menor reparo en tomar una conducta quirúrgica cuando se reinterviene por la misma vía que la inicial, debido a la utilización de las mismas incisiones. Kwak y colaboradores11 demostró que existe mayor tendencia a reoperar en forma laparoscópica a aquellos pacientes que habían sido sometidos a una cirugía laparoscópica inicialmente y viceversa, para aquellos que habían sido operados por laparotomía en forma inicial. En nuestra serie hubo mayor tendencia a reoperar en forma laparotómica a aquellos pacientes que habían sido convertidos en la cirugía inicial, y cuando se lo comparó con el grupo 1 se objetivó una diferencia estadísticamente significativa.

Adicionalmente un impedimento importante para poder reoperar por vía laparoscópica es la dilatación de asas generada por un cuadro de peritonitis generalizada. Al reoperar en forma más precoz se asume que la dilatación es menor, permitiendo realizar la cirugía laparoscópica. Esto también se traduce en una tasa no despreciable de conversión en los pacientes reoperados por vía laparoscópica, cuyo principal impedimento fue que la gran distensión de asas dificultaba el abordaje. En nuestra serie la tasa de conversión de los pacientes reoperados por vía la- paroscópica fue del 8,23\%, lo cual podría deberse a una adecuada selección de pacientes y a una reintervención precoz.

En nuestra serie, la táctica quirúrgica más utilizada es la confección de una ostomía proximal a la dehiscencia, habiéndose realizado en el $87 \%$ de los casos. Dicha estrategia es factible cuando no existe alteración de la vascularización de los cabos anastomosados y cuando el defecto es menor al 50\%. En caso contrario se debe desmontar la anastomosis y confeccionar una ostomía terminal. La ventaja de una ostomía lateral radica principalmente en la menor complejidad del segundo tiempo. La morbilidad de una reconstrucción de una ostomía lateral es notablemente menor cuando se la compara con una reconstrucción de una ostomía terminal, por ejemplo, operación de Hartmann. Adicionalmente las tasas de ostomías definitivas son menores cuando la ostomía es lateral. ${ }^{18}$

La disminución en el promedio de la estadía hospitalaria obedece seguramente a que se mantienen ciertos beneficios del abordaje miniinvasivo cuando se reopera al paciente por vía laparoscópica, si bien no hubo diferencias estadísticamente significativas. En nuestra publicación anterior ${ }^{27} \mathrm{y}$ en las de varios autores ${ }^{11,16,17}$ también se evidenció una menor estadía hospitalaria en los pacientes operados por vía laparoscópica.

La tasa de mortalidad de los pacientes que presentaron dehiscencia anastomótica fue del 6,6\%, sensiblemente menor a varias publicaciones. Marres y col. ${ }^{14}$ describen una serie de 127 pacientes, incluyendo pacientes con manejo conservador. Nueve de 44 pacientes que debieron ser reoperados fallecieron en el postoperatorio, una tasa del $25 \%$. En dicha serie muchos pacientes fueron reoperados en forma convencional, lo que podría tener relación con una elevada mortalidad.

\section{Limitaciones}

El trabajo presentado es retrospectivo, por lo tanto, existe una clara selección de pacientes dependiendo del estado general del paciente y del criterio del cirujano. Es lógico pensar que los pacientes más comprometidos sistémicamente o con un cuadro abdominal más complejo fueron reoperados en forma convencional y eso puede impactar en la evolución postoperatoria, independientemente de la vía de abordaje.

\section{CONCLUSIONES}

La reintervención laparoscópica es un tratamiento válido y seguro para el manejo de la dehiscencia anastomótica en cirugía laparoscópica colorrectal. 


\section{BIBLIOGRAFÍA}

1. Salem JF, Gummadi S, Marks JH. Minimally invasive surgical approaches to colon cancer. Surg Oncol Clin N Am 2018;2:303-18.

2. Chadi SA, Fingerhut A, Berho M, et al. Emerging trends in the etiology, prevention, and treatment of gastrointestinal anastomotic leakage. J Gastrointest Surg 2016;20: 2035-51.

3. Feo LJ, Jrebi N, Asgeirsson T, et al. Anastomotic leaks: technique and timing of detection. Am J Surg 2014;207:371-4.

4. Rotholtz N, Bun M, Lencinas S, Malizia P, Iovaldi M, Mezzadri N. Laparoscopic approach for complicated diverticulitis. Dis Colon Rectum 2005;48:668-76.

5. Rotholtz N, Laporte $\mathrm{M}$, Lencinas $\mathrm{S}$, et al. Complicaciones en cirugía colorrectal laparoscópica. Rev Argent Cirug 2007;93: 222-34.

6. Arnell TD. Minimally invasive reoperation following laparotomy. Clin Colon Rectal Surg 2006; 19:223-27.

7. Boyce SA, Harris C, Stevenson A, Lumley J, Clark D. Management of low colorectal anastomotic leakage in the laparoscopic era: More than a decade of experience. Dis Colon Rectum 2017; 60: 807-14.

8. Chang KH, Bourke MG, Kavanagh DO, Neary PC, O'Riordan JM. A systematic review of the role of re-laparoscopy in the management of complications following laparoscopic colorectal surgery. Surgeon 2016; 14: 287-93.

9. Cimitan A1, Contardo T, Molaro R, Morpurgo E. The role of laparoscopy in the treatment of anastomotic leaks after minimally invasive colorectal resections for cancer. Surg Laparosc Endosc Percutan Tech 2016; 26:80-4.

10. Ibañez N, Abrisqueta J, Lujan J. Reintervención tras complicaciones en cirugía colorrectal laparoscópica colorrectal. ¿Aporta ventajas el abordaje laparoscópico? Cir Esp 2018;96:109-16.

11. Kwak JM, Kim SH, Son DN, et al. J The role of laparoscopic approach for anastomotic leakage after minimally invasive surgery for colorectal cancer. J Laparoendosc Adv Surg Tech A 2011;21: 2933.

12. Lee CM, Huh JW, Yun SH, et al. Laparoscopic versus open reintervention for anastomotic leakage following minimally invasive colorectal surgery. Surg Endosc 2015;29: 931-6.

13. Marano A, Giuffrida MC, Giraudo G, Pellegrino L, Borghi F. Management of peritonitis after minimally invasive colorectal surgery: can we stick to laparoscopy? J Laparoendosc Adv Surg Tech A 2017;27:342-47.

14. Marres CCM, van de Ven AWH, Leijssen LGJ, Verbeek PCM, Bemelman WA, Buskens CJ. Colorectal anastomotic leak: delay in reintervention after false-negative computed tomography scan is a reason for concern. Tech Coloproctol 2017;21:709-14.

15. Rosin D, Zmora O, Khaikin B, et al. Laparoscopic management of surgical complications after a recent laparotomy. Surg Endosc 2004;18: 994-96.

16. Vennix S, Abegg R, Bakker OJ, et al. Surgical re-interventions following colorectal surgery: open versus laparoscopic management of anastomotic leakage. J Laparoendosc Adv Surg Tech A 2013; 23: $739-44$.

17. Wind J, Koopman AG, van Berge Henegouwen MI, et al. Laparoscopic reintervention for anastomotic leakage after primary laparoscopic colorectal surgery. Br J Surg 2007; 94:1562-66.

18. Gessler B, Eriksson O, Angenete E. Diagnosis, treatment, and consequences of anastomotic leakage in colorectal surgery. Int $\mathrm{J}$ Colorectal Dis 2017;32:549-56.

19. Michaels AD, Mullen MG, Guidry CA, et al. Unplanned reoperation following colorectal surgery: indications and operations. J Gastrointest Surg 2017;9:1480-85.

20. Ha GW, Kim JH, Lee MR. Oncologic impact of anastomotic leakage following colorectal cancer surgery: A systematic review and meta-analysis. Ann Surg Oncol 2017;24:3289-99.

21. Turrentine FE, Denlinger CE, Simpson VB, et al. Morbidity, mortality, cost, and survival estimates of gastrointestinal anastomotic leaks. J Am Coll Surg 2015;220:195-206.

22. Are C, Talamini MA, Murata K; De Maio A. Carbon dioxide pneumoperioneum alters acute-phase response induced by lipopolysaccharide. Surg Endosc 2002;16:1464-67.

23. Evasovich M, Clark T, Horattas M, et al. Does pneumoperitoneum during laparosocopy increase bacterial translocation? Surg Endosc 1996;10:1176-79.

24. Hanly EJ, Fuentes JM, Aurora AR, et al. Carbon dioxide pneumoperitoneum prevents mortality from sepsis. Surg Endosc 2006;20:1482-87.

25. Hanly EJ, Mendoza-Sagaon M, Murata K, et al. CO2 pneumoperitoneum modifies the inflammatory response to sepsis. Ann Surg 2003;237: 343-50.

26. Horattas MC, Haller N, Ricchiutti D. Increased transperitoneal bacterial translocation in laparoscopic surgery. Surg Endosc 2003;17:1464-67.

27. Rotholtz, Laporte M, Lencinas S, Bun M, Aued L, Mezzadri $\mathrm{N}$. Is a laparoscopic approach useful for treating complications after primary laparoscopic colorectal surgery? Dis Col Rectum 2009;52:275-79.

28. Sauerland S, Agresta F, Bergamaschi R, et al. Laparoscopy for abdominal emergencies. Evidence based guidelines of the European Association for Endoscopic Surgery. Surg Endosc 2006; 20: 14-29.

\section{COMENTARIO}

El tema del trabajo y el objetivo no habían sido evaluados de esta manera a la fecha.

El grupo al que pertenecen los autores tiene reconocida experiencia y publicaciones de sus resultados en cirugía laparoscópica colorrectal, siendo de los primeros en nuestro país en comunicar sus complicaciones.

Es así que tratar el tema más álgido de la patología colorrectal, tanto en el tratamiento electivo como en la urgencia con coherencia y honestidad intelectual, valida estos resultados.

La dehiscencia anastomótica y su tratamiento resultan el punto principal de discusión en la cirugía colorrectal y su intento de profilaxis y tratamiento impresionan resultar insuficientes en la bibliografía de la especialidad. Laporte y sus colaboradores comunican sus resultados y sus complicaciones, comparando el tratamiento, tanto por cirugía laparoscópica como vía convencional y los analizan en forma comparativa. La experiencia es importante y sus resultados, incidencia son coincidentes con la literatura. Asimismo, incluye la serie histórica. El tratamiento de la dehiscencia colorrectal por vía laparoscópica implica opciones menos agresivas con mejores resultados o variables postoperatorias.

Demuestran que mejora la forma de tratamiento y las variables de estadía hospitalaria y recuperación postoperatoria al ser comparada con cirugía convencional sin aumentar el riesgo de implementar dicho abordaje. Se destaca que la tasa de mortalidad fue menor en el grupo de menor agresión. 
El trabajo expone limitaciones claras y válidas desde el punto de vista asistencial y pragmático. La conclusión es clara, concisa y válida para su implementación en su práctica diaria.

Es un trabajo con una experiencia extrapolable a la práctica clínica diaria de cualquier cirujano colorrectal.

Hugo Amarillo

Sanatorio Modelo. San Miguel de Tucumán, Tucumán, Argentina.

\section{SESIÓN 12 DE JULIO DE 2019}

Moderador: Está en discusión el tema presentado por el Servicio del Hospital Alemán. Doctor Minetti.

Dr. Miguel Minetti: Realmente, felicitaciones, Mariano. Excelente trabajo. Lo disfruté mucho. Aprendí mucho de él también y, si bien las limitantes son importantes en el trabajo, creo que la conclusión que ustedes hacen, hoy por hoy, aun en cirugía convencional, ante la presencia de una dehiscencia, es interesante. Creo que el abordaje laparoscópico tiene inconmensurables ventajas. Solamente dos cosas: la primera, a mí me parece que es importante, frente a esto, y especialmente si se trata de cirugía colónica con una anastomosis relativamente alta, uno puede llegar a resuturar. Si bien sabemos que no es la panacea, se puede llegar a resuturar y es algo más que aportaría. Y aparte de eso, nosotros le prestamos mucha importancia también al lavado terapéutico del colon: limpiar el colon a través de sondas, que sirven para ver la dehiscencia y también para lavar el colon. También es importante tener en cuenta la magnitud de la dehiscencia, no es lo mismo una dehiscencia de menos de un cuarto de la circunferencia, que una dehiscencia más grande o una dehiscencia prácticamente total. Eso respecto al colon izquierdo. Y dentro del colon izquierdo hoy también hay alguna alternativa que es interesante, que es el lavado y el empleo de la microcirugía transanal con la idea de resuturar la zona dehiscente, cuando por vía laparoscópica la grasa rectal o la grasa del mesenterio, especialmente en la cara posterior, no permite visualizarlo fácilmente. Quizá sea una alternativa también a tener en cuenta. Con respecto al colon derecho, ahí sí me quedó alguna cuestión que me gustaría preguntarte, porque el colon derecho, no sé si ustedes han hecho ileostomía previa a la dehiscencia y algún gesto de sutura o drenaje, o han abocado en forma de bicolostomía y los han incluido en los casos de abocamiento de cabos. En general, nosotros también en el lado derecho lo abordamos por vía laparoscópica si no son grandes dehiscencias, las resuturamos, las redrenamos, y lavamos la cavidad abdominal. Vuelvo a felicitar al grupo, me parece un lindo trabajo, más allá de las limitantes que lógicamente tiene, pero creo que la conclusión más importante es que creo que el abordaje laparoscópico, aun en la peritonitis generalizada, como han mostrado ustedes, un buen lavado permite realizar el tratamiento de este problema. Aun en enfermos con asas distendidas uno puede llevar a la pelvis, puede lavar el parietocólico, puede lavar los espacios subdiafragmáticos, el Morrison, que son los lugares que más frecuentemente se acumula el líquido peritonítico. Y por último, algunos de estos enfermos muchas veces en algún parietocólico hacen algún absceso que permite contemporizarlo y drenarlos por vía percutánea. No sé si ustedes han tenido que drenar por vía percutánea abscesos. Felicitaciones, nuevamente. Gracias.

Moderador: Continúa en discusión el trabajo del Hospital Alemán. ¿Algún aporte? ¿Algún comentario más? ¿Mariano?

Dr. Mariano Laporte: Dr. Minetti, muchas gracias por sus comentarios, en primer lugar quiero destacar un concepto: si operamos a los pacientes en forma precoz, el mismo paciente puede ser operado en forma laparoscópica y quizá si al mismo paciente lo dejáramos evolucionar, terminaría en la cirugía convencional. Por eso es importante destacar el abordaje temprano. Lo que comenta el Dr. Minetti de las suturas sin dudas parece ser una alternativa válida, tanto por vía endoanal como por vía abdominal, el tema es que habría que ver el origen de esta dehiscencia. No es lo mismo la dehiscencia al primero o al segundo día, que seguramente fue causada por cuestiones técnicas, que sí podía ser resuturado. Por otro lado, una dehiscencia que lleva más tiempo, noveno o décimo día por ejemplo, generalmente es motivada por una isquemia en los cabos y creo que con una rafia solamente no estaríamos resolviendo en forma satisfactoria la complicación. Por otro lado, con respecto al colon derecho, generalmente sí, si no es una dehiscencia mínima como usted dijo podemos dar un punto, pero generalmente tratamos de abocarlo. Cuando hay un defecto importante en la anastomosis no hay duda en abocar los dos cabos. Y cuando la fuga es pequeña podríamos dejar la anastomosis in situ, y hacer una ostomía proximal. Muchas gracias por los comentarios. 